\title{
INVESTIGACIONES
}

\section{El Modelo Simple de Lectura en la identificación de dificultades lectoras en educación primaria}

\author{
The Simple View of Reading in the identification \\ of reading difficulties in primary education
}

\author{
Blanca Flor Camarillo Salazar ${ }^{a}$, Gabriela Silva Maceda ${ }^{a}$ \\ Silvia Romero Contreras ${ }^{a}$ \\ ${ }^{a}$ Universidad Autónoma de San Luis Potosí, México \\ blanca_camarillo@live.com,gabysilvamac@gmail.com,romerosil@gmail.com
}

\begin{abstract}
RESUMEN
Este estudio fue realizado en una escuela primaria en San Luis Potosí, México. Tuvo tres objetivos: identificar la tipología de dificultades lectoras a partir del Simple View of Reading o Modelo Simple de Lectura (MSL); conocer la relación entre la Comprensión Lectora (CL) y la Decodificación (D), y entre la CL y la Comprensión Auditiva (CA); y determinar en qué medida las predicciones del MSL permiten identificar las dificultades lectoras. Participaron 53 alumnos de primero de primaria. Se evaluaron tres variables: CA, D y CL. Según los resultados, más de la mitad de los alumnos presentaron dificultades en D, CA o ambas. Se encontraron correlaciones estadísticamente significativas entre CL y D, y entre CL y CA. Finalmente, las predicciones del modelo fueron correctas para pronosticar las dificultades lectoras el 68\% de los casos. Se sugiere considerar al MSL como una herramienta útil para identificar las dificultades de lectura en primaria.
\end{abstract}

Palabras clave: comprensión lectora, dificultades lectoras, educación primaria.

\begin{abstract}
This study was conducted in a primary education institution in San Luis Potosí, Mexico. It had three objectives: to identify the typology of reading difficulties according to Simple View of Reading (SVR); to evaluate the relationship between Reading Comprehension (RC) and Decoding (D), as well as between RC and Listening Comprehension (LC); and to determine to what extent the SVR predictions were able to correctly identify reading difficulties. The sample consisted of 53 students in first grade. Three variables were evaluated: LC, D and RC. The results indicate that more than half of the students evaluated presented difficulties in D, LC or both. In addition, statistically significant correlations were found between RC and D, and between RC and LC. Finally, the model correctly predicted $68 \%$ of reading difficulties cases. As a conclusion, it is suggested the SVR might be considered as a useful tool in the identification of reading difficulties.

Key words: reading comprehension, reading difficulties, primary school.
\end{abstract}




\section{INTRODUCCIÓN}

Los resultados educativos de México a nivel internacional no han sido favorables. Las evaluaciones internacionales realizadas en los últimos años sitúan al país por debajo de la media esperada y, específicamente en el área de lectura, un alto porcentaje de estudiantes no alcanza un nivel básico de comprensión lectora (OCDE, 2013; OCDE, 2016). Estos resultados son aún más preocupantes si consideramos: que las dificultades de lectura tienen una influencia directa en el aprendizaje, al ser ésta un recurso de los alumnos para adquirir conocimientos (Ortega, García \& Romero, 2008); que los problemas particulares de comprensión limitan el progreso escolar de los niños que los presentan (Defior, 1993); y que ciertos estudios longitudinales señalan que los resultados educativos en los primeros años determinan, en cierta medida, los futuros logros académicos de los estudiantes (Entwisle, Alexander \& Olson, 2005).

Pese a la relevancia de favorecer las habilidades lectoras desde edades tempranas, ciertos investigadores señalan que las estrategias docentes para la enseñanza de la lectoescritura en educación básica en México son, en su mayoría, centradas en actividades de tipo mecánicas (repetir en forma escrita palabras o frases en el cuaderno o realizar ejercicios en el libro de texto, etc.), las cuales dejan a un lado el desarrollo de las funciones comunicativas del lenguaje (Pedroza, Pérez, Ramírez, Ramos \& Treviño, 2007), situación que supone limitaciones importantes para el desarrollo de la Comprensión Lectora.

Por estas razones y, aunado a la importancia de identificar y prevenir las dificultades lectoras desde las primeras fases de enseñanza, el presente estudio pretende reconocer las habilidades que influyen en el desarrollo de la lectura, tomando como referencia el Simple View of Reading o Modelo Simple de Lectura (en adelante MSL), el cual es uno de los modelos teóricos que más evidencia ha aportado en las investigaciones sobre el desarrollo de las habilidades lectoras. Asimismo, se pretende determinar la viabilidad de la concepción de este modelo para el diagnóstico de las dificultades en la comprensión en el contexto mexicano.

En este escrito, inicialmente se retomarán algunos referentes sobre el MSL para, posteriormente, exponer los resultados de un estudio realizado en la ciudad de San Luis Potosí, México, el cual tuvo tres objetivos: identificar las dificultades de los alumnos en las áreas de Decodificación y Comprensión Lingüística (algo que supondría una limitante en la Comprensión Lectora de acuerdo con el MSL); conocer la relación que existe entre la Decodificación y la Comprensión lectora, y entre la Comprensión Lingüística y la Comprensión Lectora; y, por último, determinar si es posible considerar al MSL como una primera guía para la identificación de estas dificultades.

\section{REFERENTE TEÓRICO}

\subsection{MSL Y HABILIDADES PRECURSORAS DE LA COMPRENSIÓN LECTORA}

La Comprensión Lectora es considerada como el objetivo final de la lectura (Nation, 2005; Cain, 2010), y puede entenderse como una habilidad que implica: interpretar, comprender, analizar y dar uso a textos escritos, con el propósito de adquirir significados para alcanzar metas y que el individuo pueda integrarse de manera satisfactoria en la sociedad (OCDE, 2013). Dicho de otra forma, la lectura constituye una actividad compleja que se compone 
de diversos procesos y habilidades que la hacen posible. Esta complejidad pone en evidencia la importancia de favorecer esta habilidad de forma temprana, pero más aún, la importancia de contar con modelos teóricos con una amplia evidencia en la literatura que expliquen los procesos de adquisición e identifiquen las dificultades de lectura.

En este sentido, el Simple View of Reading o Modelo Simple de Lectura MSL (Hoover \& Gough, 1990; Gough, Hoover \& Peterson, 1996) es uno de los modelos teóricos que más evidencia ha aportado en investigaciones sobre el desarrollo lector y las dificultades de lectura. Con base en este referente, la Comprensión Lectora es el resultado del desarrollo de dos habilidades precursoras: la decodificación y la comprensión lingüística.

La decodificación o reconocimiento de palabras, es entendida como la capacidad de identificar de forma precisa las palabras a partir de un texto escrito, en función de su correcta correspondencia grafema-fonema (Cain, 2010; Hoover \& Gough, 1990). En este proceso de identificación existen dos rutas de acceso al léxico: la ruta visual, y la ruta fonológica. La primera hace referencia a un reconocimiento global de las palabras que están almacenadas en el léxico mental del lector porque ya han sido procesadas anteriormente; mientras que la segunda se basa específicamente en la aplicación de las reglas de correspondencia entre grafemas y fonemas (Patterson, Marshall \& Coltheart, 1985).

En relación con los procesos de decodificación, algunos autores señalan que además de la precisión de la lectura existen otros indicadores, como la velocidad al leer, que están asociados al reconocimiento de palabras y también dan cuenta de este proceso. En este sentido, la investigación realizada en España por Serrano y Defior (2008), sugiere que, en idiomas como el español, donde la correspondencia entre grafemas y fonemas es más consistente, el factor de la velocidad de lectura es más sensible que la precisión a la hora de identificar dificultades en los procesos lectores. Sin embargo, a pesar de estos hallazgos reportados, otro estudio realizado en México sugiere que este desarrollo de la decodificación podría darse de forma distinta en el país americano, pues Silva-Maceda y Romero-Contreras (2017) encontraron, en una muestra de estudiantes de primero a cuarto grado, que la velocidad para leer un texto no es un indicador significativo por sí mismo cuando se toma en cuenta la precisión de la lectura; es decir, es posible que los estudiantes mexicanos muestren una velocidad corta para efectuar la lectura, pero esto no se traduce en la comprensión del texto escrito.

Si bien, el dominio de la Decodificación es imprescindible para que un texto sea leído, esto no garantiza la comprensión de la lectura (Jiménez-Fernández, 2014; Abusamra, Ferreres, Raiter, De Beni \& Cornoldi, 2010), lo que nos lleva al segundo componente del desarrollo lector de acuerdo con el MSL. La segunda variable importante según este modelo es la Comprensión Lingüística o Comprensión Auditiva, entendida como la capacidad de distinguir el significado de las palabras dentro de una oración y, posteriormente, integrar dichos significados para generar inferencias con un estímulo recibido de forma oral (Gough, Hoover \& Peterson, 1996). En esta habilidad inciden múltiples factores, pero entre los principales se encuentran: el conocimiento del mundo (Snow, Griffin \& Burns, 2005), la amplitud del vocabulario (Verhoeven, \& Van Leeuwe, 2008), o los conocimientos previos del tema (Hirsch, 2006). Estos aspectos a su vez son influidos por factores contextuales como las características socioeconómicas donde el alumno se desenvuelva (Hoff, 2003) y el nivel de interacción oral que se presente en el contexto del alumno (Hoff \& Naigles, 2002), entre otros. 
De esta manera, el desarrollo de la Comprensión Lectora se ilustra con la siguiente fórmula:

$$
\text { Comprensión Lectora = Decodificación x Comprensión Lingüística }
$$

De acuerdo con esta perspectiva, ninguna de las habilidades precursoras es suficiente por sí misma para que sea posible entender el significado de un texto, por el contrario, es necesario que ambas coexistan para lograr la Comprensión Lectora. Más aun, por lo que se refiere a las dificultades lectoras, el MSL considera que éstas son el resultado de la debilidad en una o dos de las habilidades precursoras (Decodificación o Comprensión Lingüística).

\subsection{TIPOLOGÍA DE DIFICULTADES DE LECTURA A PARTIR DEL MSL}

Puesto que el desarrollo de estas habilidades (Decodificación y Comprensión Lingüística) se da de forma simultánea en la mayoría de los casos, pueden existir tanto dificultades específicas como mixtas en la lectura, por lo que una tipología de dificultades de acuerdo con el MSL estaría conformada de la siguiente manera:

En primer lugar, se encuentran niños con dificultades en la Decodificación, pero con una Comprensión Lingüística adecuada. Estos niños son incapaces de leer un texto de forma fluida por lo que son frecuentemente identificados con dislexia -una dificultad inesperada en el desarrollo de la habilidad lectora en relación con la inteligencia del niño(Cain, 2010). Las manifestaciones de las dificultades en el reconocimiento de palabras implican aspectos como la precisión, la rapidez y la entonación con que se realiza la lectura (Jiménez-Fernández, 2014).

En segundo lugar, existen algunos niños con una baja Comprensión Lingüística pero una Decodificación adecuada. Aunque estos alumnos son capaces de decodificar un párrafo de forma precisa y fluida, presentan un vocabulario muy pobre que se traduce en serias limitaciones al momento de integrar el significado de un texto, por lo que con frecuencia pasan desapercibidos durante el diagnóstico de dificultades lectoras.

Por último, hay niños que presentan dificultades en ambas habilidades y que, al presentar estos dos tipos de problemas, son conocidos como lectores de comprensión débil o pobre. Cabe señalar que estos alumnos generalmente no se les considera que tengan algún trastorno específico de aprendizaje y generalmente han recibido una educación promedio a la de los demás niños.

Esta tipología de dificultades se ilustra en la Figura 1, donde se ejemplifica que los niños pueden presentar dificultades en la Decodificación, la Comprensión Lingüística, o en ambas (Hoover \& Gough, 1990; Nation, 2005; Cain, 2010). 
Figura 1. Tipología de las dificultades de lectura, tomado de: Silva-Maceda (2013).

\section{Comprensión Lingüística}

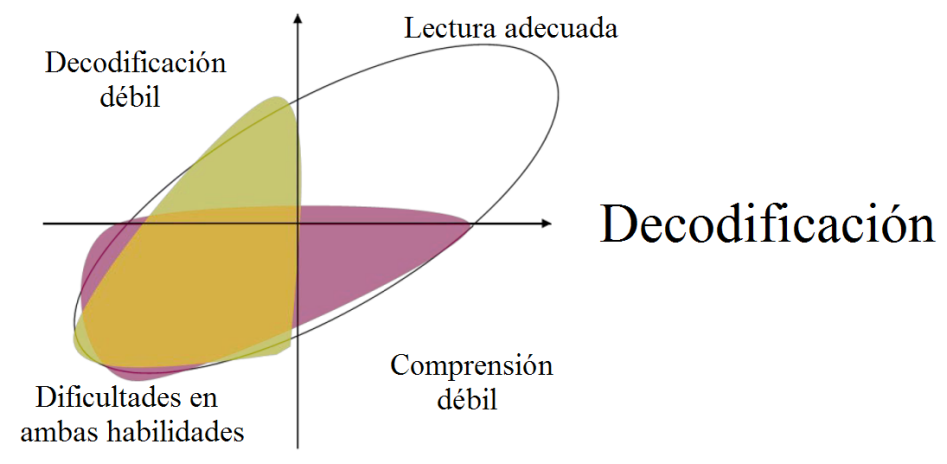

De acuerdo con la categorización anterior, será importante reconocer que cada alumno puede presentar un tipo de limitación distinto, por lo que requerirá de apoyos y recursos diferentes para hacer frente a sus dificultades.

\subsection{PATRÓN DE DESARROLLO DE LA COMPRENSIÓN LECTORA}

Por lo que se refiere al patrón de desarrollo de la Comprensión Lectora propuesto por el MSL, la evidencia muestra que, en el idioma inglés, la Decodificación presenta una correlación más fuerte con la lectura en los primeros años de educación formal, y que es hasta quinto grado de primaria, cuando el lector alcanza la automaticidad en el reconocimiento de palabras, que la Comprensión Lingüística o Auditiva empieza a tener preponderancia sobre la Decodificación. En concreto, un meta-análisis realizado por Gough, Hoover y Peterson en 1996, mostró que las correlaciones entre la Decodificación y la Comprensión Lectora eran más fuertes en el primero y segundo ciclo de enseñanza $(r=.61)$ a diferencia de la Comprensión Auditiva durante el mismo periodo $(r=.41)$. Posteriormente, a partir del quinto grado escolar, estos coeficientes disminuían considerablemente en el caso del reconocimiento de palabras y aumentaban en el caso de la Comprensión Auditiva. Este patrón de desarrollo es ilustrado en la Figura 2. 
Figura 2. Correlaciones entre la Decodificación y la Comprensión Auditiva con la Lectura, del meta-análisis de Gough, Hoover \& Peterson (1996). Fuente: Elaboración propia.

\section{Correlaciones entre la Decodificación y Comprensión Auditiva con la Lectura}

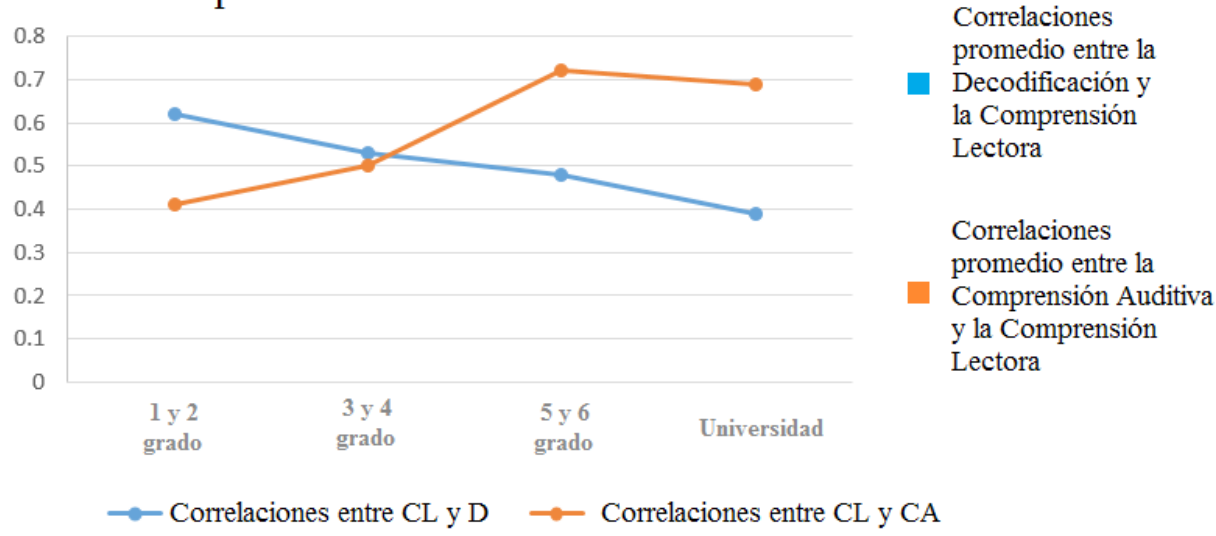

Aunque los resultados encontrados por Gough y colaboradores suponen este cambio en la preponderancia de cada variable precursora con el transcurrir de la educación formal, ciertos estudios sugieren que este patrón de desarrollo pudiera darse de forma distinta en el idioma español pues, al tratarse de un idioma más transparente, donde las correspondencias entre grafemas y fonemas son más consistentes, las habilidades de Decodificación son más fáciles de adquirir que en el idioma inglés.

\subsection{IDENTIFICACIÓN DE DIFICULTADES LECTORAS}

Con respecto a la identificación de las dificultades lectoras, la utilización del MSL como punto de partida ha sido comúnmente documentada en el idioma inglés. Por mencionar algunos estudios, Catts y Weismer (2006) evaluaron a un total de 182 alumnos, y concluyeron que los estudiantes que presentaron dificultades en la Comprensión Lectora también presentaban frecuentemente limitaciones en el área de Comprensión Lingüística (aun habiendo desarrollado una Decodificación adecuada); señalaron, además, que el MSL cuenta con ventajas sobre otros modelos teóricos que se centran únicamente en el reconocimiento de palabras o la comprensión de la lectura. De modo similar, Catts, Herrera, Corcoran y Bridges (2015), midieron la influencia de la Decodificación y la Comprensión Lingüística sobre la Comprensión Lectora, y encontraron que las habilidades precursoras presentaban diferencias significativas en alumnos con y sin dificultades lectoras. Así pues, dedujeron que los componentes del MSL deben incluirse en las evaluaciones de detección temprana diseñadas para identificar a estudiantes con dificultades en la comprensión.

Por el contrario, las investigaciones realizadas con base en el MSL en un idioma más transparente como lo es el español (donde las correspondencias grafema-fonema son más directas) son menos frecuentes. Uno de los estudios realizados en torno a estas dificultades 
en nuestro idioma fue llevado a cabo en Chile por Infante, Coloma y Himmel (2012), y buscó determinar la influencia de la Decodificación y Comprensión Oral o Lingüística sobre la Comprensión Lectora. Dentro de los aportes principales, encontraron que la Comprensión Lingüística tiene una incidencia importante en la Comprensión Lectora solo en aquellos casos donde la decodificación es adecuada o promedio. Más aún, los resultados sugirieron que un rendimiento adecuado en el reconocimiento de palabras y la Comprensión Lingüística no asegura la Comprensión Lectora, caso contrario a lo que sucede en las investigaciones en el idioma inglés.

Aunque los estudios anteriormente mencionados muestran un patrón de desarrollo con implicaciones para la enseñanza de la lectura, no es posible generalizarlos al contexto mexicano, especialmente por el hecho de que el español tiene características distintas a las del inglés o -en el caso de la investigación realizada en español- las características de los sistemas educativos podrían tener impactos diferentes. Por esta razón, es conveniente efectuar investigaciones que evalúen la influencia de los componentes del MSL en un idioma transparente, en el contexto social y educativo particular de cada país, para evaluar su factibilidad como una herramienta en la identificación de las dificultades de lectura.

\section{MÉTODO}

La presente investigación se realizó bajo un enfoque cuantitativo, de muestra transversal, y de tipo descriptivo. Tuvo tres objetivos: el primero, identificar la tipología de dificultades de lectura a partir del MSL en una muestra de niños mexicanos de una escuela primaria pública; el segundo, conocer la relación existente entre la Decodificación y la Comprensión lectora, y entre la Comprensión Lingüística y la Comprensión Lectora en alumnos del primer ciclo de primaria; $y$, el tercero, determinar en qué medida el MSL predice de manera correcta las dificultades lectoras en los alumnos.

\subsection{PARTICIPANTES}

Para la composición de la muestra, se realizó un muestreo no probabilístico (por conveniencia) para un total de 53 estudiantes de entre 6 y 7 años, de primer grado de educación básica en una institución pública de la ciudad de San Luis Potosí, México. A cada padre de familia se le envió una carta de consentimiento informado donde se explicó el objetivo del estudio y las actividades requeridas para la participación del alumno. La carta iba acompañada de un breve cuestionario sociodemográfico. Sólo aquellos alumnos cuyos padres dieron su autorización participaron en las actividades para la recolección de información.

\subsection{MATERIALES}

Para la recolección de los datos se utilizaron tres subpruebas estandarizadas para medir diferentes variables.

Para evaluar la Comprensión Lingüística o Auditiva se utilizó la subprueba Entendiendo Párrafos, de CELF 4 - Spanish, (Semel, Wiig \& Secord, 2006). La CELF 4-Spanish es una batería de evaluación psicométrica diseñada para evaluar el desarrollo del lenguaje en personas de 5 a 21 años, con normas de la población de habla hispana en Estados Unidos. 
Específicamente en la subprueba Entendiendo Párrafos, el objetivo es determinar en qué medida un alumno comprende (a nivel literal e inferencial) una serie de textos que son leídos en voz alta. Su aplicación es de forma individual, en una sola sesión. Para efectos de este estudio, se utilizó la puntuación directa, pues las puntuaciones escalares provenientes de la norma sólo tenían sentido en combinación con el resto de las subpruebas de la batería y diferían en sus valores mínimo y máximo dependiendo del rango de edad.

Para el caso de la Decodificación, se utilizó la subprueba Lectura de Pseudopalabras del Test de Lectura y Escritura en Español LEE (Defior-Citoler, Fonseca, Gottheil, Aldrey, Pujals, Rosa \& Serrano, 2006), que es una batería de evaluación orientada a psicopedagogos, psicólogos educativos e investigadores que puede aplicarse a niños de $1^{\circ}$ a $4^{\circ}$ grado para identificar su riesgo de desarrollar dificultades en torno a la lectura y escritura. La subprueba utilizada consistió en mostrar al alumno una serie de palabras inventadas, las cuales debían ser leídas en voz alta por el alumno, mientras que se registra la precisión en el reconocimiento de las pseudopalabras y el tiempo empleado para realizar la actividad (velocidad de lectura). Cabe señalar que esta prueba tiene como objetivo evaluar la mera habilidad de decodificación en su forma más pura pues, al leer palabras inventadas, el alumno no cuenta con recursos léxicos que le faciliten el reconocimiento de las grafías, por lo que tiene que recurrir a su conocimiento de las correspondencias entre sonidos y grafías (o también llamada ruta fonológica) para poder descifrar el escrito.

Por último, para conocer el nivel de Comprensión Lectora se utilizó la subprueba Comprensión de Textos de la misma batería de pruebas LEE, cuyo propósito es medir la habilidad de un alumno para comprender tres textos diferentes, dos narrativos y uno expositivo. Esta comprensión se evalúa a partir de preguntas de tipo literal e inferencial, así como la capacidad de identificar frases que representen el título del texto leído o que lo resuman de forma adecuada.

\subsection{PROCEDIMIENTO}

Las evaluaciones se realizaron de forma individual con cada niño afuera del salón de clases y en una sola sesión. Con cada uno se estableció rapport y se procedió a la aplicación de las pruebas.

\section{RESULTADOS}

Primero, se reportan los estadísticos descriptivos del desempeño de la muestra en habilidades precursoras, así como en la Comprensión Lectora. En los casos de las pruebas Precisión de Lectura de Pseudopalabras, Velocidad de Lectura de Pseudopalabras y Comprensión de Textos de la batería LEE, se consideraron las puntuaciones percentiles obtenidas por los alumnos puesto que la batería ofrece un listado de baremos que permite calcular la posición relativa del niño con respecto a una norma. En el caso de la subprueba Entendiendo Párrafos, de CELF 4 Spanish no fue posible calcular dichas medidas estandarizadas puesto que la batería solo ofrece baremos a partir de una aplicación completa de la prueba, por lo que se consideraron las puntuaciones directas, las cuales corresponden a un valor que va de 0 a 15. Como se ilustra en la siguiente tabla, la puntuación promedio en la variable Comprensión Lingüística se ubicó en un valor de $\bar{x}=10.09$; mientras que la media en Comprensión Lectora fue de $\bar{x}=33.08$. 
Tabla 1. Estadísticos descriptivos para puntuaciones de las habilidades de Decodificación, Comprensión Auditiva y Comprensión Lectora $(\mathrm{N}=53)$

\begin{tabular}{|l|c|c|c|c|}
\hline & Media & Desviación estándar & Mínimo & Máximo \\
\hline Edad (años) & 6.47 & .50 & 6 & 7 \\
\hline Entendiendo párrafos & 10.09 & 2.09 & 4 & 14 \\
\hline Lectura de Pseudopalabras & 19.06 & 16.06 & 1 & 90 \\
\hline Comprensión de textos & 33.08 & 27.69 & 1 & 90 \\
\hline
\end{tabular}

En relación con los datos anteriores, es de considerar que el rendimiento en las pruebas de Precisión de Lectura de Pseudopalabras y Comprensión de Textos presentan un promedio por debajo del percentil 50 y además muestran una variabilidad alta en los datos ( $s=16.06$ y $s=27.69$, respectivamente), lo que indica que existen alumnos con una alta habilidad de reconocer palabras y comprender textos, pero a su vez alumnos con serias dificultades para realizar dichas tareas. En relación con la estimación para Entendiendo Párrafos, es posible observar que el promedio de la muestra corresponde a un valor de $\bar{x}=10.09$, de un rango posible que va de 0 a 15, así como una desviación estándar de $s=2.09$; sin embargo, considerando que estas puntuaciones no representan medidas estandarizadas (como es el caso de los percentiles), no es posible aseverar que el desempeño corresponda o no al esperado en esta muestra.

Para atender al objetivo principal de este estudio, el cual consistió en identificar a los alumnos que presentaron dificultades en las habilidades precursoras (Decodificación y Comprensión Lingüística); se elaboró un diagrama de dispersión simple con ambas variables. En el caso de la Decodificación, se consideraron las puntuaciones percentiles en la prueba de Precisión en Lectura de Pseudopalabras, mientras que, en el caso de la Comprensión Linguiística, el valor considerado fue la puntuación directa de la prueba Entendiendo Párrafos. Conviene subrayar que, para este diagnóstico de dificultades, los puntajes obtenidos en la prueba Velocidad de Lectura de Pseudopalabras no fueron considerados, esto con base en los hallazgos encontrados por Silva-Maceda y RomeroContreras (2017) en un estudio realizado con una muestra mexicana. En el diagrama de dispersión se definieron los siguientes puntos de corte:

Para clasificar a los alumnos a partir de su desempeño en Decodificación, se delimitó un punto de corte del percentil 25 atendiendo a la recomendación citada en la batería LEE, la cual establece que un puntaje igual o por debajo de esta puntuación representa un indicador de riesgo alto de presentar dificultades en el área evaluada, en este caso la Decodificación. Para el caso de la Comprensión Lingüística, se definió un punto de corte de -1 desviación estándar a partir de la media obtenida por la muestra, lo cual, para este caso, corresponde a un valor de $7.99=8$. En otras palabras, un niño con una valoración igual o menor a estas estimaciones se clasificó como con dificultades en esa habilidad particular y un alumno con una puntuación mayor fue considerado como competente.

A partir de la distribución anterior, se identificaron 32 niños con lo que, de acuerdo con el MSL, representaría una dificultad únicamente en la Decodificación de palabras; 1 con dificultades solo en la Comprensión Auditiva; y 9 niños con ambos tipos de dificultades. En contraste, 11 de los alumnos fueron clasificados como buenos lectores (ver Figura 3). 
Figura 3. Tipología de las dificultades de lectura según las puntuaciones obtenidas en cada una de las habilidades precursoras del MSL.

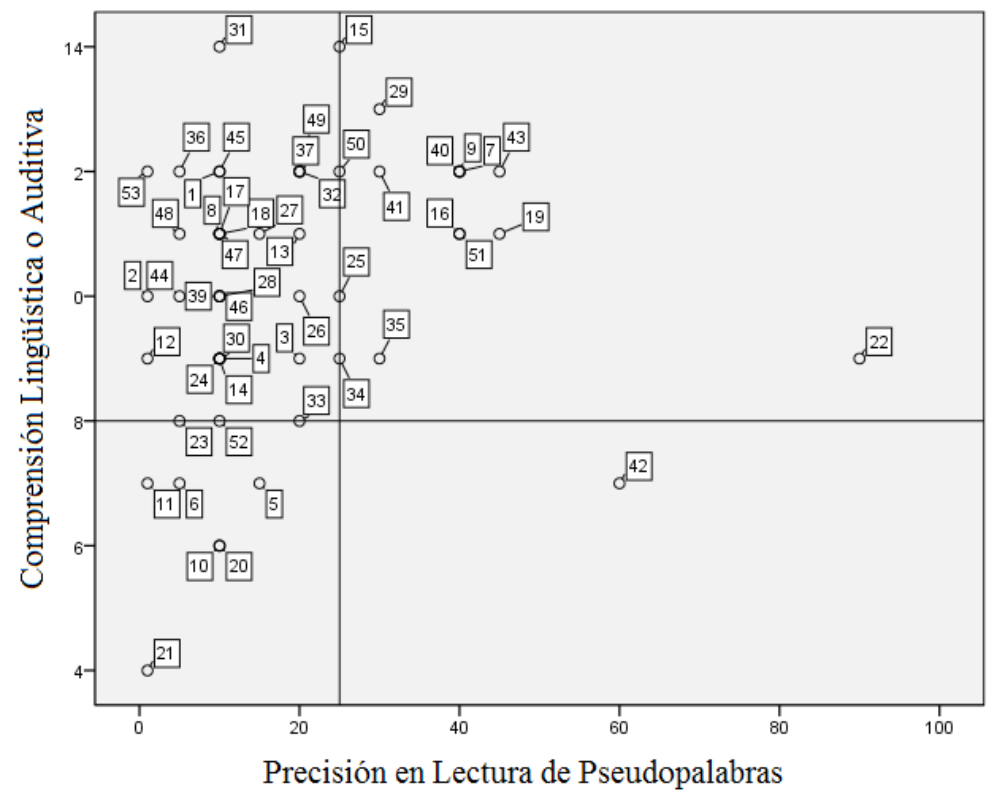

Como se observa en la Figura 3, más de la mitad de los alumnos que componen la muestra presenta algún tipo de dificultad en una habilidad precursora específica o en ambas, lo que, de acuerdo con el MLS, se traduciría en una limitación para el desarrollo de la Comprensión Lectora. Es posible notar que una de las áreas donde los alumnos muestran mayor dificultad es la Decodificación, es decir, muchos estudiantes han alcanzado un nivel de comprensión oral que les permite entender los textos que escuchan, pero no son capaces de leerlos de forma precisa. Aunque esto pareciera explicarse por el hecho de que los niños se encuentran adquiriendo el principio alfabético, hay que recordar que el desempeño en el área es limitado en comparación con la norma establecida para estudiantes del mismo grado.

Para atender el segundo objetivo de esta investigación, es decir, conocer la relación que existe entre la Decodificación y la Comprensión Lectora, y entre la Comprensión Auditiva y la Comprensión Lectora en esta muestra, se calculó el coeficiente de correlación de Pearson para datos paramétricos, el cual tiene como propósito determinar si existe una relación lineal entre dos variables. Se obtuvieron las relaciones promedio entre cada variable precursora y la Comprensión de textos y se encontró una correlación estadísticamente significativa, alta, entre la Decodificación y la Comprensión Lectora $(r=.78, p=<.001)$. En el caso de la Comprensión Auditiva y la Comprensión lectora, se obtuvo una correlación mediana, también estadísticamente significativa $(r=.35, p=<.009)$. Estos resultados apoyan a los encontrados por Gough y colaboradores en 1996, donde se sugiere que, en los primeros años de enseñanza, la habilidad para reconocer las palabras impresas tendrá mayor peso a la hora entender el significado de lo escrito. A continuación, estos resultados son contrastados con los encontrados en 1996: 
Tabla 2. Coeficientes de correlación encontrados en este estudio en comparación con los reportados en la literatura

\begin{tabular}{|l|c|c|}
\hline & $\begin{array}{l}\text { Coeficientes reportados } \\
\text { en este estudio. Primer } \\
\text { ciclo de primaria }\end{array}$ & $\begin{array}{l}\text { Coeficientes reportados por Gough, } \\
\text { Hoover \& Peterson en 1996. Primer } \\
\text { y segundo ciclo de primaria }\end{array}$ \\
\hline $\begin{array}{l}\text { Decodificación y la Compren- } \\
\text { sión Lectora }\end{array}$ & $.78^{* *}$ & $.61^{* *}$ \\
\hline $\begin{array}{l}\text { Comprensión Auditiva y Com- } \\
\text { prensión Lectora }\end{array}$ & $.35^{* *}$ & $.41^{* *}$ \\
\hline
\end{tabular}

Como puede observarse en la tabla anterior, los coeficientes reportados en el presente estudio presentan un comportamiento similar a los observados en el meta-análisis citado y, aunque el papel de la Decodificación parece estar más asociado a la comprensión de lo escrito, no es posible asumir que el patrón de desarrollo de la Comprensión Lectora se esté dando de forma similar en el español que en el idioma inglés, pues es necesario contar con datos comparables en años posteriores de la educación primaria.

Finalmente, para atender el tercer y último objetivo del estudio, es decir, determinar en qué medida el MSL predice correctamente las dificultades de lectura en esta muestra, se realizó una estimación de para cuántos casos las dificultades o bien la competencia en la Decodificación, Comprensión Lingüística o ambas, correspondía con las dificultades o competencia en la variable Comprensión Lectora. Considerando que el MSL considera indispensable ser competente en ambas habilidades precursoras para alcanzar la comprensión de un texto, se determinó que el tener una dificultad en al menos una de ellas constituiría un predictor de dificultades en la comprensión. En el sentido contrario, un desarrollo adecuado de la Decodificación y la Comprensión Auditiva implicaría el pronóstico de una Comprensión Lectora competente.

Para determinar si un alumno era considerado como Con dificultades o Competente, se respetaron los mismos puntos de corte que en el análisis anterior, asignando a su vez el valor del percentil 25 para el caso de la prueba Comprensión de Textos. Se analizó caso por caso, para identificar si el alumno era competente o no en las habilidades precursoras y comprobando si su pronóstico de acuerdo con el MSL se cumplía en relación con su Comprensión Lectora. Para esta muestra, las predicciones del modelo fueron correctas en 36 de los casos (68\%), es decir, las dificultades en una o ambas habilidades precursoras correspondían a dificultades en la Comprensión Lectora; asimismo, la competencia en ambas correspondía a ser un lector competente. Las predicciones del modelo no fueron acertadas en 17 casos (32\%). De los alumnos en que las predicciones no fueron cumplidas, en 16 niños las habilidades precursoras predecían que fueran lectores con dificultades y no lo eran; mientras que en el caso restante el modelo predecía que fuera un lector competente y no se cumplió el supuesto. 


\section{DISCUSIÓN}

De acuerdo con los resultados encontrados, es posible observar que un alto número de los alumnos evaluados no alcanza un nivel óptimo en las habilidades de Decodificación y Comprensión Lingüística, variables que, de acuerdo con el MSL, son necesarias para que se alcance una Comprensión Lectora adecuada.

El área donde se presentan las mayores dificultades corresponde a la Decodificación, donde un alto porcentaje de los alumnos presentaron puntuaciones bajas. Estos resultados no apoyan los hallazgos encontrados por Treviño et al. (2007) en cuanto a que las habilidades de Decodificación se ven más favorecidas que la Comprensión Lectora en las estrategias utilizadas por docentes en aulas mexicanas. De acuerdo con estas hipótesis, esperaríamos encontrar un desempeño más alto en la Decodificación (Precisión de Lectura) que en las pruebas de Entendiendo Párrafos y Comprensión de Textos. Sin embargo, en relación con esta discrepancia es pertinente considerar dos cuestiones, las cuales son explicadas a continuación.

En primer lugar, aunque el rendimiento en la subprueba Entendiendo Párrafos es observablemente alto, es preciso señalar que una limitación encontrada en esta medición es no contar con normas estandarizadas que ofrezcan un panorama del desempeño del alumno respecto a la norma establecida con una muestra mayor, por lo que su punto de comparación se sitúa solamente en relación con sus compañeros de clase. Además, ciertos estudios previos han encontrado discrepancias en la medición de la Comprensión Lingüística en muestras similares a las de este estudio que pudieran estar asociadas a esta limitante. Tal es el caso del estudio realizado por Silva-Maceda y Camarillo (2016a) el cual concluyó que los reactivos de la subprueba de CELF 4 - Spanish (Entendiendo Párrafos) podrían resultar relativamente fácil en su ejecución para los alumnos de $1^{\circ}$ a $4^{\circ}$ grado, lo cual se traduce en un desempeño alto generalizado en la prueba y un bajo rendimiento solo en aquellos casos con un riesgo significativamente alto de presentar dificultades en el área de comprensión oral. Para esta investigación, lo anterior supondría que algunos alumnos de esta muestra pudieran presentar dificultades en la Comprensión Lingüística, pero no han sido identificados porque, aunque respecto a la norma podrían mostrar limitaciones, respecto a sus compañeros muestran un rendimiento aceptable.

En segundo lugar, el hecho de que las puntuaciones en la Decodificación sean representativamente bajas pone de manifiesto el hecho de que, aunque las prácticas docentes puedan estar centradas en la enseñanza del reconocimiento de palabras en los primeros años (Treviño et al., 2007), no es posible asegurar que dichas prácticas sean las ideales para que el alumno aprenda las reglas de correspondencia grafema-fonema. Para apoyar este supuesto habrá que decir que, durante la aplicación de las pruebas, se observó que algunos estudiantes son capaces de leer de forma correcta palabras que son conocidas para ellos (por ejemplo, en la subprueba Comprensión de Textos), en contraposición a lo que sucede en la lectura de palabras inventadas o pseudopalabras, donde los niños presentan dificultades. Estas observaciones también han sido reportadas con anterioridad pues se ha encontrado una mayor facilidad por parte del alumno para leer de forma correcta y fluida palabras que les son familiares en comparación con aquellas que han visto por primera vez (Silva-Maceda \& Camarillo, 2016b). Bajo este contexto, se hace evidente que los alumnos de esta muestra recurren principalmente a la ruta léxica y no a la fonológica para la identificación de las palabras, un indicativo de que las reglas de correspondencia grafema-fonema no han sido aprendidas totalmente. 
En relación con el segundo objetivo de este estudio, el cual fue conocer las relaciones entre las habilidades precursoras de la lectura (Decodificación y Comprensión Auditiva) y la Comprensión lectora y su relación con el patrón de desarrollo propuesto por el MSL, cabe señalar que los resultados encontrados en esta muestra coinciden con la hipótesis de que, en años iniciales de educación formal, la habilidad para leer palabras tendrá una correlación alta con la habilidad para dar sentido a un texto escrito, y que será hasta edades posteriores cuando la Comprensión Auditiva tendrá un mayor peso a la hora de comprender un texto. Aunque estas similitudes son sugestivas, vale la pena recordar que, como limitación de este estudio, la presente muestra no consideró la evaluación a estudiantes de grados posteriores en educación básica, por lo que no es posible asumir que el desarrollo de las habilidades precursoras (y su influencia en la Comprensión Lectora) se estén dando de forma equivalente al observado en el idioma inglés.

Del mismo modo, en relación con el tercer objetivo que fue determinar en qué medida el MSL predice las dificultades lectoras en los alumnos, los presentes resultados permiten sugerir que el MSL proporciona una primera guía para la evaluación de las habilidades que impactan en la Comprensión Lectora en los primeros años; lo anterior debido a que un porcentaje significativo de las predicciones del modelo fueron correctas para esta muestra. Los resultados de estas predicciones reafirman lo propuesto por Catts, Herrera, Corcoran y Bridges (2015) acerca de que es pertinente considerar componentes del MSL en las evaluaciones de detección temprana de las dificultades específicas en la Comprensión. Esta evidencia constituye una herramienta valiosa, ya que idealmente pueden evaluarse estas habilidades en las primeras etapas de aprendizaje con el propósito de identificar las dificultades específicas que cada alumno presenta y, de esta manera, brindar una atención orientada a las necesidades particulares de cada estudiante.

Si bien estos hallazgos resultan valiosos en el campo de la investigación, habrá que tener en cuenta algunas limitaciones en la realización de este estudio. En primer lugar, es de considerar que el tamaño de la muestra es reducido y el tipo de muestreo utilizado no permite efectuar predicciones ni aplicaciones del modelo a otras poblaciones. Para este caso, será necesario efectuar investigaciones más amplias que permitan tener un panorama más certero sobre las implicaciones del uso del MSL en la detección temprana de dificultades lectoras. En segundo lugar, es necesario involucrar más instrumentos estandarizados para la evaluación de cada variable, en vez de utilizar sólo una subprueba, esto para minimizar el riesgo de que algunos resultados se vean influidos por las características del instrumento y no correspondan a las habilidades auténticas del alumno. Será importante, además, que dichos instrumentos cuenten con medidas estandarizadas que permitan la comparación del desempeño en cada habilidad respecto a una norma general, contrario a lo que sucede en el caso de la subprueba Entendiendo Párrafos.

\section{CONCLUSIONES}

De acuerdo con lo expuesto en las secciones anteriores, es posible considerar algunas conclusiones importantes.

En primer lugar, un número significativo de alumnos en esta muestra presentaron dificultades, al menos en una de las variables precursoras de la comprensión lectora, situación que pone de manifiesto las limitaciones en el desarrollo de esta habilidad entre 
los estudiantes mexicanos, y que a su vez se exponen en diversas evaluaciones nacionales e internacionales. En relación con este punto, resulta necesario realizar una detección temprana de estas dificultades y ofrecer a los alumnos estrategias e intervenciones pertinentes que les permitan mejorar su competencia lectora.

En segundo lugar, las correlaciones entre las habilidades precursoras de la lectura y la comprensión de textos permiten estimar que, en primer grado de escolarización, la Decodificación presenta una asociación más fuerte con la Comprensión Lectora, a diferencia de la Comprensión Auditiva, cuya relación se da en menor intensidad. Aunque estos resultados presentan similitudes con el patrón inicial de desarrollo de la comprensión propuesto por el MSL, no es posible asumir que esta evolución se dé en forma similar en grados posteriores. No obstante, si es posible visibilizar las variables que inciden mayormente sobre la comprensión desde las etapas más tempranas, y las implicaciones que esto supondría para la enseñanza de la lectura.

En tercer lugar, es posible considerar que los supuestos teóricos del MSL son pertinentes en la identificación de dificultades lectoras en nuestro idioma, ya que un alto porcentaje de predicciones en esta muestra fueron correctas a la hora de determinar las dificultades en la comprensión de textos de los alumnos. Sin embargo, será necesario realizar estudios más amplios para explorar esta viabilidad en otros grados y contextos escolares.

Finalmente, aunque el presente trabajo constituye un primer paso hacia la identificación de dificultades específicas en áreas de lectura en un idioma como el español, resulta imprescindible realizar más investigaciones que se centren en conocer los procesos de desarrollo de la Comprensión Lectora en nuestro idioma, esto con la finalidad de aportar a la mejora de la enseñanza de esta habilidad desde los primeros años escolares.

\section{REFERENCIAS BIBLIOGRÁFICAS}

Abusamra, V., Ferreres, A., Raiter, A., De Beni, R. \& Cornoldi, C. (2010). Leer para comprender: test para la evaluación de la comprensión de textos.

Cain, K. (2010). Reading development and difficulties, (Vol. 8). John Wiley \& Sons.

Catts, H., Herrera, S., Nielsen, D. \& Bridges, M. (2015). Early prediction of reading comprehension within the simple view framework. Reading and Writing, 28(9), 1407-1425.

Catts, H. \& Weismer, S. (2006). Language deficits in poor comprehenders: A case for the simple view of reading. Journal of Speech, Language, and Hearing Research, 49(2), 278-293.

Defior, S. (1993). Las dificultades de lectura: papel que juegan las deficiencias del lenguaje. Comunicación, Lenguaje y Educación, 5(17), 3-14.

Defior-Citoler, S., Fonseca, L., Gottheil, B., Aldrey, A., Pujals, M., Rosa, G. \& Serrano, F. (2006). LEE. Test de lectura y escritura en español. Psicología y Psicopedagogía, 6(17).

Entwisle, D. R., Alexander, K. R. \& Olson, L. S. (2005). First grade and educational attainment by age 22: A new story. American Journal of Sociology, 110(5), 1458-1502.

Gough, P., Hoover, W. \& Peterson, C. (1996). Some observations on a simple view of reading. Reading comprehension difficulties: Processes and intervention, 1-13.

Hirsch, E. D. (2006). The knowledge deficit. Houghton Mifflin Harcourt, ISBN-10, 618657312.

Hoff, E. (2003). The specificity of environmental influence: Socioeconomic status affects early vocabulary development via maternal speech. Child development, 74(5), 1368-1378.

Hoff, E. \& Naigles, L. (2002). How children use input to acquire a lexicon. Child development, 73(2), 418-433. 
Hoover, W. \& Gough, P. (1990) The simple view of Reading. Reading and writing: An Interdisciplinary Journal, 2(2), 127-160.

Infante, M., Coloma, C. J. \& Himmel, E. (2012). Comprensión lectora, comprensión oral y decodificación en escolares de $2^{\circ}$ y $4^{\circ}$ básico de escuelas municipales. Estudios pedagógicos (Valdivia), 38(1), 149-160

Jiménez-Fernández, G. (2014). Las dificultades de aprendizaje: una aproximación didáctica. Editorial Avicam. Granada (España).

Nation, K. (2005). Children's Reading comprehension difficulties. The Science of Reading: a Handbook. Vol. 9 John Wiley \& Sons.

OCDE (2013). Programme for International Student Assessment 2012. Volumen II.

(2016). Resultados de PISA 2015. En OCDE. Recuperado de: https://www.oecd.org/pisa/ PISA-2015-Mexico-ESP.pdf

Ortega, N., García, R. \& Romero, M. (2008) Reconceptualizando a la lectura como recurso de aprendizaje. Revista Científica Electrónica de Psicología, (6), 40-64.

Patterson, K., Marshall, J., \& Coltheart, M. (1985). Surface dyslexia: Cognitive and neuropsychological studies of phonological reading. London: LEA.

Pedroza, H., Pérez, G., Ramírez, P., Ramos, G. \& Treviño, G. (2007). Prácticas docentes para el desarrollo de la comprensión lectora en primaria. Instituto Nacional para la Evaluación de la Educación.

Semel, E. M., Wiig, E. H. \& Secord, W. (2006). CELF 4: clinical evaluation of language Fundamentals. Pearson: Psychological Corporation.

Serrano, F. \& Defior, S. (2008). Dyslexia speed problems in a transparent orthography. Annals of dyslexia, 58(1), 81 .

Silva-Maceda, G. (2013). Are better communicators better readers? An exploration of the connections between narrative language and reading comprehension (Tesis doctoral). Universidad de Wolverhampton, Inglaterra.

Silva, G. \& Camarillo, B. (2016a). Identificación de las dificultades de lectura en niños de primaria. Presentación, Celaya, Guanajuato.

. (2016b). Relación entre Velocidad de Lectura y Comprensión Lectora en alumnos de primaria. Presentación, Cd. Obregón, Sonora.

Silva-Maceda, G. \& Romero-Contreras, S. (2017). Leer rápido no siempre es igual a comprender: Examinando la relación entre velocidad y comprensión. Revista Costarricense de psicología, 36(2), 123-144.

Snow, C., Griffin, P. \& Burns, M. S. (2005). Knowledge to support the teaching of reading: Preparing teachers for a changing world. John Wiley \& Sons.

Treviño, E., Pedroza, H., Pérez, G., Ramírez, P., Ramos, G. \& Treviño, G. (2007). Prácticas docentes para el desarrollo de la comprensión lectora en primaria. Instituto Nacional para la Evaluación de la Educación.

Verhoeven, L. \& Van Leeuwe, J. (2008). Prediction of the development of reading comprehension: A longitudinal study. Applied Cognitive Psychology, 22(3), 407-423. 
